\title{
EXPERIENCIAS DE ESTUDIANTES SOBRE LA CRISIS SOCIOSANITARIA EN CHILE: DESAFÍOS PARA EL SISTEMA EDUCACIONAL
}

\author{
HeRman Pezo Hoces ${ }^{1}$ \\ Claudia Castilla García ${ }^{2}$ \\ PAULINA CASTILLO ${ }^{3}$ \\ YADIRA PALENZUELA FUNDORA ${ }^{4}$ \\ CAROLINa Álvarez ${ }^{5}$ \\ CAMILA ARAYA ${ }^{6}$ \\ YULIET CRUZ MARTÍNEZ ${ }^{7}$ \\ PABLO MARTÍNEZ CASTRO ${ }^{8}$ \\ SEBASTIÁN ESCOBAR GONZÁLEZ ${ }^{9}$
}

\begin{abstract}
RESUMEN
El artículo presenta los avances de una investigación en curso que se propone aportar a la comprensión de las experiencias de estudiantes de instituciones educativas municipales y subvencionadas en el contexto de crisis sociosanitaria en Chile. Desde un enfoque cualitativo, se produjo información a partir de entrevistas a catorce jóvenes estudiantes, chilenos/as y extranjeros/as de diferentes regiones y comunas del país, con el objetivo de dar cuenta de una diversidad de realidades. El trabajo finaliza con algunas reflexiones acerca de los desafíos que enfrenta el sistema educativo en contextos de cambios y crisis social, a partir de la propia experiencia estudiantil.
\end{abstract}

PALABRAS CLAVES: ESTUDIANTES, CRISIS, ESCUELA, EXPERIENCIAS.

${ }^{1}$ Trabajador social y Magíster@ en Sociología Universidad Alberto Hurtado. Integrante del Núcleo de Investigación y Acción en Juventudes, Universidad de Chile. Correo electrónico: hermanfabian.pezo@gmail.com

${ }^{2}$ Psicóloga; Máster en Teoría y Metodología de las Ciencias Sociales; Doctora en Ciencias Sociales. Integrante del Núcleo de Investigación y Acción en Juventudes, Universidad de Chile. Correo electrónico: claudiacastillagarcia@gmail.com

${ }^{3}$ Psicóloga; Magíster en Ciencias Sociales mención Sociología de la Modernización, Universidad de Chile. DoctoraC Universidad Autónoma de Barcelona. Integrante del grupo de Investigación Globalisation, Education and Social Policies, UAB. Núcleo de Investigación y Acción en Juventudes, Universidad de Chile. Correo electrónico: paulinacastillo.h@gmail.com

${ }^{4}$ Psicóloga y Máster en Psicología Educativa, Universidad de La Habana, Cuba. Magíster en Análisis Sistémico Aplicado a la Sociedad y Doctora en Psicología por la Universidad de Chile. Integrante del Núcleo de Investigación y Acción en Juventudes, Universidad de Chile y Miembro de GT Infancia y Juventudes de CLACSO. Correo electrónico: ypalenzuela@gmail.com

${ }^{5}$ Licenciada en Historia; Magíster en Ciencias Sociales de la Universidad de Chile; Doctora $\odot$ en Educación, Universidad Alberto Hurtado-Universidad Diego Portales. Integrante del Núcleo de Investigación y Acción en Juventudes, Universidad de Chile. Correo electrónico: caroalvarezvaldes@gmail.com

${ }^{6}$ Cientista Política; Magíster en Métodos para la Investigación Social, Universidad Diego Portales. Investigadora del Núcleo de Investigación y Acción en Juventudes, Universidad de Chile. Correo electrónico: carayaguzman@gmail.com

${ }^{7}$ Psicóloga; Máster en Psicología Social y Comunitaria, Universidad de La Habana. Estudiante de Doctorado en Ciencias Sociales, Universidad de Chile, becaria ANID. Integrante del Núcleo de Investigación y Acción en Juventudes, Universidad de Chile. Correo electrónico: mariposa17981@gmail.com

${ }^{8}$ Sociólogo, Universidad de Chile. Integrante del Núcleo de Investigación y Acción en Juventudes, Universidad de Chile. Correo electrónico: pablo.martinez@ug.uchile.cl

${ }^{9}$ Licenciado en Educación, Pontificia Universidad Católica de Chile, y Magíster en Ciencias Sociales, Universidad de Chile. Académico de la Facultad de Educación de la Universidad Finis Terrae y miembro del Núcleo de Investigación y Acción en Juventudes, Universidad de Chile. Correo electrónico: siescobar@uc.cl 


\title{
EXPERIÊNCIAS ESTUANTES DA CRISE SOCIAL E SANITÁRIA NO CHILE: DESAFIOS PARA O SISTEMA EDUCACIONAL
}

\begin{abstract}
RESUMO
O trabalho se propõe a contribuir para a compreensão das experiências de alunos de instituições de ensino municipais e subsidiadas no contexto da crise social e sanitária no Chile. A partir de uma abordagem qualitativa, as informações foram produzidas a partir de entrevistas com 14 jovens estudantes, chilenos e estrangeiros, de diferentes regiões e comunas do país, a fim de dar conta de uma diversidade de realidades. O trabalho termina com algumas reflexões sobre os desafios enfrentados pelo sistema educacional em contextos de mudança e crise social, a partir da própria experiência do aluno.
\end{abstract}

PALAVRAS-CHAVE: ESTUDANTIS, CRISE, ESCOLA, EXPERIÊNCIA.

\section{STUDENTS' EXPERIENCES OF THE SOCIAL AND HEALTH CRISIS IN CHILE: CHALLENGES FOR THE EDUCATIONAL SYSTEM}

\begin{abstract}
The work proposes to contribute to the understanding of the experiences of students from municipal and subsidized educational institutions in the context of the social and health crisis in Chile. From a qualitative approach, information was produced from interviews with 14 young students, Chilean and foreign, from different regions and communes of the country, in order to account for a diversity of realities. The work ends with some reflections about the challenges faced by the educational system in contexts of change and social crisis, based on the student's own experience.
\end{abstract}

KEYWORDS: STUDENTS, CRISIS, SCHOOL, EXPERIENCE. 


\section{INTRODUCCIÓN Y PROBLEMATIZACIÓN}

A fines de 2019, a partir de la Revuelta social iniciada el 18 y 19 de octubre en Chile (Araujo, 2019; Cuevas y Budrovich, 2021; Garcés, 2020), la mayoría de los colegios y universidades estuvieron en paro de actividades por la situación nacional, mientras el Ministerio de Educación (MINEDUC) enviaba orientaciones a las instituciones educativas para afrontar las consecuencias ante las pérdidas de clases y el retraso de los cronogramas de actividades curriculares durante semanas. Fue en este contexto, que el curso escolar del 2019 culmina flexibilizando sus actividades y postergando para el siguiente año el cumplimiento de otras que no pudieron llevarse a cabo.

Marzo de 2020 comienza con un nuevo reto. Con la llegada del Covid-19 al país y la rápida propagación del virus a escala nacional, se declara estado de excepción constitucional de catástrofe, que perdura hasta hoy. Con ello llegan medidas de confinamiento estricto y las escuelas cesan sus clases presenciales en marzo, a solo dos semanas de haber iniciado el nuevo curso escolar. Este nuevo período de crisis, por motivos sociosanitarios, da comienzo a transformaciones inéditas para las cuales no estaban preparadas las instituciones educativas: la virtualización de la educación. En términos de recursos, el MINEDUC realiza una serie de acciones fundamentalmente dirigidas a instituciones municipales $\mathrm{y} / \mathrm{o}$ subvencionadas para contribuir $\mathrm{y} / \mathrm{o}$ garantizar herramientas tecnológicas que permitieran llevar a cabo la formación a distancia. Así, se realizan convenios con empresas tecnológicas y se ponen en marcha plataformas de colaboración de 
educación o docencia virtual ${ }^{10}$, entre otros. En términos de normativas y protocolos se identifican tres ejes predominantes: a) dimensión curricular de los procesos educativos para garantizar la efectividad y continuidad de la educación a pesar del impacto del contexto; b) procesos de cuidados, convivencias, relaciones afectivas y salud mental; y en estrecha relación con este último, los que se centran en c) la protección, control y seguridad de los/as actores educativos. El término «flexibilizar» adquiere notoriedad en todos los protocolos y orientaciones (MINEDUC, 2019, 2020e), denotando quizás uno de los cambios más medulares que ha tenido que enfrentar el sistema educativo producto de la pandemia.

Esta necesidad de flexibilizar el currículum y los procedimientos producto de la situación coyuntural pone en tensión las arraigadas tradiciones de estandarización y disciplinamiento y abre un posible camino para la transformación. Con la llegada de la pandemia se observa en los protocolos mayormente un refuerzo de la contención emocional (MINEDUC, 2020d), la comprensión, el diálogo y la comunicación (MINEDUC, 2020a, 2020e), y la búsqueda del bienestar emocional (MINEDUC, 2020b, 2020e). El ámbito de protección, control y seguridad de los/as actores educativos está relacionado con la responsabilidad y la ética del cuidado (MINEDUC, 2020a) y explícitamente con orientaciones y protocolos que permitan comprender la escuela como entorno de protección (MINEDUC, 2019). Sin embargo, transversalmente, en toda la documentación analizada, podemos evidenciar una mirada adultocéntrica (Duarte, 2018), debido a los procesos participativos limitados que se expresan en

\footnotetext{
${ }^{10}$ Se realizan convenios con grandes empresas tecnológicas como Microsoft y Google. A su vez, se pusieron en práctica diversas plataformas educativas como: «Aprendo en Línea», «TV Educa Chile», «Desarrollo Docente en línea», «Biblioteca Digital Escolar», «Tutores para Chile», por citar las más relevantes. Además, se efectuaron otras acciones como otorgar accesos a internet a establecimientos del país con subsidio estatal, «Becas TIC JUNAEB», entrega de tablet a estudiantes, ofrecer preuniversitarios online gratuitos y becas para prueba de transición. Para el curso escolar 2021, se implementó la plataforma «Sigamos Aprendiendo».
} 
acciones y recomendaciones a las instituciones educativas. En este sentido, todas las normativas se centran en el cuidado, educación y protección de los/as diferentes actores, particularmente las/os estudiantes, pero ubican el peso y responsabilidad de esta misión en el mundo adulto y dentro de este, con mayor peso, en los/as actores directivos de las instituciones educativas (MINEDUC, 2020e). La pandemia trajo un reto —o una amenaza si se lee en clave de pérdida de poder - para las instituciones educativas, al tener que traspasar responsabilidades del proceso formativo curricular a las familias y a las y los estudiantes (MINEDUC, 2020a). Ello es una transformación que, sin lugar a duda, requerirá ser analizada en el tiempo, a partir de su permanencia o no, y sus impactos en toda la concepción del modelo educativo.

A pesar de estos esfuerzos (MINEDUC, 2020a, 2020d, 2020e), el alcance de muchas de estas medidas no es experimentada directamente por las y los estudiantes, quienes desde realidades familiares diversas, con situaciones socioeconómicas distintas a lo largo del país y en especial en zonas rurales, han tenido que hacer frente a estos cambios principalmente con apoyos de las propias instituciones educativas, o desde estrategias familiares, teniendo que compartir recursos y espacios del hogar para tales fines con otros miembros de su familia. Por otra parte, no se cuenta con mayores antecedentes de investigación a nivel nacional que hagan referencia a cómo han experienciado estos períodos de crisis las y los jóvenes, y cuáles han sido los significados en torno a estos hitos de la historia reciente del país.

Es ante este escenario que nos propusimos comprender las experiencias de estudiantes de instituciones educativas municipales y subvencionadas en el contexto actual de crisis en Chile, para apuntar con estos resultados algunas reflexiones y retos para pensar las instituciones educativas en contextos de cambios y crisis social, dando centralidad a la experiencia de las y los estudiantes. La estructura de este texto cuenta con un primer apartado donde se discuten los 
principales antecedentes de investigación relacionados a cómo se observan y construyen las juventudes en los contextos educativos, así como el lugar de la escuela en contextos de crisis. A continuación, se ponen en diálogo estos referentes con los hallazgos de la investigación, para cerrar con algunas ideas integradoras.

\section{RUTAS TEÓRICAS PARA COMPRENDER LA ESCUELA EN TIEMPOS DE CRISIS}

Las instituciones educativas se han estructurado como ámbitos de socialización formal por excelencia (Lahire, 2004), con un currículum, didácticas, tiempos y secuencias que no solo buscan transmitir conocimientos, sino que normas y valores (Dubet, 2013). Sin embargo, ellas son más que sus currículums y sus funcionamientos. Una línea predominante concibe a la escuela como una institución clave en la reproducción del orden social (Foucault, 2012), destacando que encierran relaciones de poder y dominación que se reproducen y perpetúan en la sociedad. Aunque estas lógicas no son inamovibles, dicho cambio constituye un desafío y requiere observar la escuela como un espacio que es posible transformar, distanciándose de toda lectura determinista que no vea posibilidades o alternativas de cambio.

En este sentido, una apuesta es ampliar el prisma desde el cual se comprende la educación; esta requiere ser entendida como espacio clave para promover el cambio social, más allá de sus límites formales, más allá de una institución con lógicas establecidas, para pensarse como una comunidad educativa que puede enriquecer y potenciar las relaciones que en estos espacios tienen lugar, tomando en cuenta la vida cotidiana de las y los sujetos que la integran, sus contextos de existencia y lo que es fundamental, los modos en que 
sus miembros experiencian e internalizan los procesos educativos (Fariñas, 2005).

En una línea similar, otros autores plantean ver la escuela revelando los imaginarios de lo que es considerado aprendizaje (Foladori et al., 2014). Este proceso al cual el autor denomina «aulear», implica una manera activa de transitar por el encuentro en las relaciones (profesor-estudiante/estudianteestudiante) donde no interesan tan solo los resultados: «aulear como instalación de condiciones de posibilidad para que algo allí se dé, lo que habrá de visualizarse en un cambio de ritmo del proceso mismo» (Foladori et al., 2014: 15). Por su parte, Simons y Masschelein (2014) se preguntan sobre qué es lo escolar y defienden el tiempo de la escuela, como un tiempo no productivo que implica un espacio-tiempo de pensar y salirse de la cotidianidad tan dominada por la familia, una apertura a otro lugar — la escuela como lugar—. Además, debe tomar en cuenta la relevancia de los momentos biográficos y los contextos situados en los que se producen, pues el aprendizaje es experiencial y se da desde dinámicas de interrelación (Dubet y Martuccelli, 1997).

En este sentido, nos resulta valiosa también la propuesta acerca de la construcción del conocimiento sobre la escuela y la educación desde el Enfoque Histórico Cultural EHC (Vygotski, 1987), relevando la importancia del proceso educativo más allá de la mirada tradicional como institución que provee una educación centrada en la transmisión y acumulación de conocimientos y poniendo el foco en la relación enseñanza-aprendizaje. La escuela debe ser vista como el espacio potencial en que múltiples redes de relaciones pueden contribuir al desarrollo personal de los miembros de la comunidad educativa, donde la educación no se limita solamente a los espacios formales, sino también a su articulación con otros espacios relacionales como la «educación familiar, a la educación de sentido común presente en las relaciones de la vida cotidiana, a la educación que tiene lugar a través de los medios de comunicación, la 
autoeducación, entre otras» (Fariñas, 2005: 46; Foladori et al., 2014) y el papel que la educación juega en la sociedad, como motor de cambio.

Por todo ello, es central para esta investigación la categoría experiencia. Siguiendo a Joan Scott (2001), los sujetos no tienen la experiencia, sino que son constituidos por medio de ella. De ese modo, puede decirse que poner de relieve la experiencia de las juventudes es establecer su identidad como personas con agencia. Desde esta perspectiva, la experiencia no es lo que debemos evidenciar desde la investigación social, sino más bien aquello que se busca explicar.

En esta cuerda, al decir del sociólogo Francois Dubet, «el análisis de la experiencia social es útil, no para describir lo "vivido" de los individuos, sino para comprender cómo se producen nuestras maneras de vivir juntos» (Dubet, 2011: 129). De modo que, si bien uno de los significados de la noción de experiencia se refiere al flujo de emociones, sentimientos e ideas, acá la entenderemos más bien, siguiendo a Dubet $(2010,2011)$, como una actividad de los individuos para construir el sentido de sus prácticas a partir de una heterogeneidad de principios constitutivos.

El acontecimiento se constituye en experiencia a través de la elaboración subjetiva, que en definitiva se produce a partir del bagaje cultural y social con que cuenta cada individuo (Araujo, 2009) y se hace accesible a los demás a través del discurso (Martuccelli y Santiago, 2017; Scott, 2001).

A partir de ello, nuestra propuesta implica explorar el vínculo entre los individuos y el sistema educativo en tanto dimensión estructural de la sociedad, partiendo de la subjetividad. Por ello, la pregunta acerca de cómo en el presente escenario de crisis sociosanitaria, las experiencias vividas impactan las aspiraciones de las y los jóvenes, resulta fundamental, entendiendo que estas son «lógicas sociales que orientan el quehacer de los actores en relación a sus alternativas y posibilidades» (Sepúlveda y Valdebenito, 2014: 6) y se configuran 
a partir de los contextos históricos concretos de producción al ser depositarias de una cultura internalizada (Appadurai, 2004). Estas aspiraciones se producen dentro de un contexto de crisis y transformación y específicamente dentro de la escuela se están dando transformaciones en cuanto a las relaciones de autoridad con el mundo adulto, poniendo en crisis modelos hegemónicos donde las y los adultos tenían el conocimiento y, por ende, el poder con respecto a las y los estudiantes (Neut, 2015). Igualmente se identifica la existencia de una ruptura entre el currículum y las relaciones, aquello que el estudiante experiencia cuando va a la escuela y las relaciones que se dan en espacios presenciales más allá de la sala de clase (Dussel, 2020). Todo ello se da dentro de un contexto de conflicto $\mathrm{y}$ resistencia del mundo adulto, pero donde igualmente surgen espacios antihegemónicos en las escuelas y distintas formas de resistencias estudiantiles (Pezo, 2019). Sin embargo, esta resistencia estudiantil se sitúa en la periferia de la construcción programada del conocimiento, al margen del aprendizaje supuestamente principal, pasando así al terreno del currículo oculto y del aprendizaje indirecto (Fariñas, 2005). Todo ello se agudiza por el contexto de una profunda crisis social y sanitaria, donde las y los docentes deben cumplir con las orientaciones establecidas por la institucionalidad, en situaciones emocionales y socioeconómicas diversas, al tiempo que desempeñarse en un lenguaje virtual que es mayormente habitado por las generaciones más jóvenes, entregar el seguimiento y control de los procesos educativos a las familias y flexibilizar todo un proceso tradicionalmente marcado por la inflexibilidad (Dussel, 2020).

La transformación no solo de las condiciones materiales, sino del propio vínculo y relación pedagógica en la virtualidad, marcado por las dinámicas de inclusión/exclusión y desigualdades sociales, los cambios y experiencias de las y los profesores durante este tiempo, así como la poca información que se tiene sobre las experiencias de participación de las y los estudiantes en las decisiones 
sobre cómo afrontar la nueva situación, hacen que el presente estudio alcance relevancia. De esta manera, comprender las experiencias de estudiantes de instituciones educativas municipales y subvencionadas en el contexto actual de crisis en Chile contribuirá a reflexionar acerca de los desafíos que enfrenta el sistema educativo actualmente, desde un posicionamiento que rescata la centralidad estudiantil y sus experiencias como eje fundamental desde el cual enfrentar la crisis; vistos no como pasivos receptores de las políticas educativas, sino como actores protagónicos.

\section{RUTA METODOLÓGICA}

La presente investigación se aborda desde un enfoque metodológico cualitativo (Creswell, 2015), el cual busca producir conocimientos relacionados con las experiencias de las y los estudiantes de instituciones educativas municipales y subvencionadas en el contexto de crisis social y sanitaria en Chile. Para ello utilizamos el muestreo teórico (Strauss y Corbin, 2002), a partir de cinco criterios de selección: a) el tipo de establecimiento educativo: municipal y subvencionado — para observar cómo se vive la pandemia en instituciones que inicialmente no contaban con recursos para la formación a distancia y sus relaciones con las orientaciones ministeriales_-; b) cursos de interés ( $8^{\circ}$ básico, $1^{\circ}, 2^{\circ}, 3^{\circ}$ y $4^{\circ}$ medio), donde se pueden develar momentos biográficos claves de cambios en su devenir como estudiantes o en toma de decisiones sobre su futuro escolar; c) género, para analizar las posibles diferenciaciones que se producen desde las construcciones culturales en torno al género, tomando en cuenta también las evidencias que ya van señalando un mayor impacto de la pandemia sobre las mujeres en términos de sobrecarga en los cuidados, aumento de la violencia, entre otros; d) presencia de estudiantes extranjeros/as en la muestra para favorecer una diversidad de experiencias, y e) diversidad de comunas y representación de algunos casos fuera de la Región Metropolitana para indagar 
en particularidades del fenómeno en distintos contextos. De esta manera, la intencionalidad de la muestra busca dar cuenta de la diversidad a partir de diferentes indicadores.

La técnica de producción de información utilizada fue la entrevista semiestructurada (Trindade, 2014), entendiéndose como una interacción online producto de la pandemia - guiada por objetivos establecidos en función de la investigación, realizada a partir de un guion de temas, pero dejando libertad de orden y respuestas a las y los entrevistados; se empleó como criterio para cerrar la muestra, la saturación de la información (Strauss y Corbin, 2002).

La muestra final estuvo conformada por catorce jóvenes (64\% mujeres y el $35 \%$ hombres) entre 12 y 18 años, quienes se encontraban estudiando en los niveles primarios y secundarios de escolarización (entre $8^{\circ}$ básico y $4^{\circ}$ medio). La mayoría reside en la Región Metropolitana, en comunas que permiten contar con una diversidad de realidades (La Cisterna, Recoleta, Maipú, Ñuñoa, Independencia, Padre Hurtado, Peñalolén), pero también hay jóvenes de otras regiones como Ñuble, Los Lagos, Los Ríos, así como de diversas nacionalidades como Chile, Colombia, Cuba y Haití. La estrategia de análisis estuvo orientada por el enfoque de análisis de contenido, entendido como la observación, interpretación y producción de información, a partir de un conjunto de procedimientos «que tienen como objetivo la producción de un meta-texto analítico en el que se representa el corpus textual de manera transformada» (Díaz y Navarro, 1995: 181). 


\section{EXPERIENCIAS DE LAS Y LOS ESTUDIANTES EN RELACIÓN}

CON LA ESCUELA, LOS PROCESOS EDUCATIVOS Y LAS

RELACIONES AFECTIVAS EN EL CONTEXTO DE CRISIS

SOCIOSANITARIA CHILENA

Las experiencias de las y los estudiantes con relación a las transformaciones de los procesos educativos en el contexto revuelta-pandemia fueron analizadas en cinco dimensiones: a) la experiencia de las clases a distancia; b) las condiciones materiales y soportes institucionales con los que cuentan para enfrentar este nuevo escenario; c) las relaciones con docentes, compañeros/as y familia; d) las aspiraciones futuras; y e) las prácticas de cuidado/autocuidado.

En general, las y los estudiantes dan cuenta de un primer momento, cuando se cierran las escuelas y comienzan las medidas más restrictivas a propósito de la pandemia, de la poca claridad en las orientaciones y toma de decisiones respecto al modo de continuar con las clases y su educación. Este primer tiempo, con la esperanza de una corta duración de estas medidas, fue más bien de desarrollar tareas en casa, por medio de guías y material pedagógico. En este sentido, afirman que hubo una mala organización del liceo, información mal distribuida y que estuvieron varios meses a la deriva hasta que el colegio decidió hacer las clases online. Este punto es particularmente destacado por estudiantes de enseñanza básica.

Sí, nosotros la verdad este año recién empezamos a implementar las clases online, así como más estrictamente, porque el año pasado como nadie sabía muy bien lo que iba a pasar, $\mathrm{mmm}$, los profes tampoco tenían una forma de estudio a distancia, entonces se fueron acomodando a la situación y su solución como colegio fue guías y nosotros aprender de ellas y hacerlas, y hacer ejercicios, y la explicación. (E12, $\mathrm{H}, 1^{\circ}$ medio, RM, Lago Ranco)

Ello reafirma cuán poco estaban preparadas las instituciones y todo el sistema educativo, para una transformación de tamaña envergadura. A pesar de 
los protocolos de MINEDUC para enfrentar la situación, se evidencia un desfase entre estos y el alcance en la práctica docente, al menos desde la experiencia de las y los estudiantes. Ello no puede desconocer, al mismo tiempo, las limitaciones de las y los docentes para manejar las herramientas necesarias para una práctica virtual, especialmente quienes se desempeñan en establecimientos públicos (CIAE, 2020).

En cuando a las experiencias de las clases online predomina la apreciación de que con esta modalidad el aprendizaje es muy limitado, que aprenden menos en comparación con las clases presenciales. Esto porque les cuesta más aprender y si hay cosas que no entienden, las dudas no se aclaran con rapidez, como sí ocurre en la modalidad presencial.

No aprendo mucho, o sea, pa mí este es un año perdido, uno va a pasar a cuarto medio sin saber nada. (E2, F, $3^{\circ}$ medio, RM, Recoleta).

La percepción sobre la dificultad de las clases online y las labores asociadas las relacionan con el exceso de tareas, cansancio y sobrecarga; ello pone en cuestionamiento el énfasis en las normativas acerca de evitar que esto ocurra (MINEDUC, 2020a, 2020d, 2020e), al tiempo que es coherente con el mayor peso puesto en garantizar lo curricular en el proceso (MINEDUC, 2020a, 2020d, 2020e). Esta vivencia de sobrecarga en las y los estudiantes no puede entenderse al margen de la disminución de lo relacional como base fundamental de lo curricular (Foladori et al., 2014).

La experiencia de las y los estudiantes con relación al poco aprendizaje curricular durante este período es confirmada por recientes estudios del propio MINEDUC (2021), en los que se muestra que el $60 \%$ de los estudiantes entre $6^{\circ}$ básico y $4^{\circ}$ medio no alcanzaron durante el 2020 los aprendizajes necesarios, y nueve de cada diez estudiantes de $3^{\circ}$ y $4^{\circ}$ medio experimentan haber sido afectados en sus aprendizajes (MINEDUC, 2021). A lo anterior se suma el 
aburrimiento, la distracción durante las clases y las dificultades de concentración en esta modalidad, expresada por las y los estudiantes de nuestro estudio; en estrecha relación, está la dificultad para organizar los tiempos.

A pesar de lo anterior, las y los estudiantes reconocen algunos aprendizajes o ganancias de este proceso, básicamente relacionadas con el manejo de las tecnologías, de herramientas informáticas para las clases y las evaluaciones. Algunos/as estudiantes comentan que ahora tienen más autonomía producto de este nuevo formato de clases.

Personalmente, la pandemia me ayudó mucho, he aprendido a ser autodidacta, a tener un plan de estudio, me gusta que no haya un gendarme diciendo que tienes que estudiar y hacer tal cosa. Nos debieron haber educado así desde un principio, a ser autodidactas. (E8, M, $4^{\circ}$ medio, RM, Independencia)

Ello requiere situarse al menos en dos cuestiones. En primer lugar, el formato pedagógico virtualizado, en ciertos aspectos es más cercano a estudiantes, a sus habilidades y prácticas. En segundo lugar, la relación entre la flexibilización de los procesos educativos (MINEDUC, 2019; MINEDUC, 2020e), la disminución del control docente en esta modalidad (MINEDUC, 2020a) y el aumento de la vivencia de la autonomía en las y los estudiantes mostrado en nuestras entrevistas. Ello sin duda nos conduce a pensar en vías que van mostrando cómo transformar las tradiciones educativas que impiden una participación del estudiantado en todos los procesos educativos que los involucran, que democraticen las lógicas de saber-poder tradicionales en las prácticas escolares.

La modalidad de clases virtuales exige ciertas condiciones materiales para enfrentarlas de buena manera. $\mathrm{Al}$ respecto, a pesar de que se observan problemáticas comunes y transversales para garantizar un acceso a la educación de calidad, cabe señalar que, en su mayoría, estos requerimientos se resuelven de manera individualizada, a partir de las estrategias que elaboran cada estudiante 
con sus familias. En este sentido, varios/as estudiantes señalan tener dificultades en conectividad; carencia de aparatos tecnológicos en buen estado o suficientes: asistir a clases con el celular (suyo o de un familiar); falta de espacio adecuado, en el que puedan estar sin interferencias o interrupciones. Igualmente, algunos/as estudiantes afirman tener las condiciones materiales requeridas. Las desigualdades en las condiciones para enfrentar la docencia online han sido confirmadas por otras investigaciones (Fundación 99, 2020) y puede derivar en ciertos riesgos en la permanencia y continuidad de estudios:

Yo considero que es horrible, hueón. Considero que de verdad es súper penca. Tengo que despertarme, subir un cerro con una silla cagao de frío a sentarme en la hueá y verla en un celu y ni siquiera tengo una mesa, entonces tengo que escribir como en mi pierna po, como en el cuaderno en la pierna y fin. Y si se me acaba la batería, si quiero ir al baño tengo que bajar el cerro, que se me cae la conexión y subirlo de nuevo, entonces hay algunas veces que bajo y digo «no, sabí que, no subo más» porque de verdad que me da flojera. (E14, M, $3^{\circ}$ medio, RM, Casa Blanca/Padre Hurtado)

En menor medida, algunos/as estudiantes señalan que desde sus instituciones educativas han recibido algunos soportes en particular, facilitando la entrega de chips y recursos tecnológicos que permitan acceder a las clases. No obstante, se argumenta que esta entrega no ha sido garantizada universalmente. Los datos evidencian esta brecha ampliamente (Fundación 99, 2020).

La dimensión relacional del proceso de educación a distancia fue abordada también. Respecto a ello, destaca uno de los estudiantes que, si bien reconoce que las instituciones educativas se han esforzado para adecuar las estrategias de enseñanza-aprendizaje ante este escenario, ello no ha contemplado de manera suficiente una preocupación por el factor socioemocional:

El liceo no se ha preocupado de la parte psicológica de los estudiantes, se ha preocupado más que nada de la parte del aprendizaje, y eso está mal, siento que es súper importante, 
súper fundamental, porque al tener las cosas claras en ese tipo de temas se puede aprender bien. (E12, F., $1^{\circ}$ medio, RM, Lago Ranco)

Nuevamente se evidencia la distancia entre lo que se norma — donde se dio espacio a las cuestiones más afectivas y relacionales-y la práctica. Estos hallazgos resultan contrastantes con una de las conclusiones de un estudio que encuestó a 2.205 docentes de todo el país, cuando afirma que la pandemia «ha relevado la gran preocupación de las/los docentes por el aspecto scioemocional de sus alumnos/as» (CIAE, 2020: 22). Lo anterior apunta hacia una ruptura con lo esencial del proceso educativo: el vínculo relacional y el soporte socioemocional que significa finalmente la escuela. La pregunta de qué manera la escuela interviene como un espacio de contención y protección durante la crisis, surge inevitablemente; nuestros datos parecieran indicar un proceso débil en este aspecto.

Los datos brindados por estudios del propio MINEDUC alertan al señalar que durante el 2020: «A nivel socioemocional, 70\% de los niños, niñas y jóvenes dijo tener dificultades para expresar sus emociones; y el 45\% de los niños y niñas de alta vulnerabilidad señaló que les gustaría que los profesores les preguntaran cómo ha sido estar lejos del colegio. La cifra se acentúa en los escolares de $4^{\circ}$ básico, donde llega a un 71\%» (MINEDUC, 2021).

La importancia de esta esfera relacional se reafirma cuando se observa que las y los estudiantes declaran que uno de los aspectos que más extrañan de esta modalidad es la vinculación directa entre pares, con los cuales poder compartir y relacionarse; incluso en algunos casos se expresa como miedo a no volver a ver a los/as amigos/as. También manifiestan que esta modalidad dificulta las interacciones entre estudiantes durante las clases.

Así, plantean que las relaciones que han mantenido más cercanas son con sus familiares directos y amistades. En algunos casos se habla de relaciones con 
profesores de establecimientos y en menor medida, pareja u otro vínculo. Respecto a las relaciones con familiares, estas se dan principalmente con padres, madres y otros familiares con los que viven. Estas relaciones, en algunos casos, se señala que se han fortalecido, y que la familia ayuda a organizarse en los estudios; en tanto, en otros se señalan conflictos, principalmente por organización de espacios para trabajar o estudiar y en otros, por conflictos preexistentes.

Nos organizamos y ya no hay pelea, no hay tanta pelea, y na po, la relación con mi familia todo bien, o sea, perfecto. (E1, F, $4^{\circ}$ medio, RM, La Cisterna)

Las manifestaciones de conflicto son coherentes con investigaciones que señalan cómo la niñez y juventud se ve vulnerada al tener un tiempo prolongado de encierro, y específicamente al estar expuestos al estrés de las familias y posibles situaciones de violencia intrafamiliar, situaciones que podrían agudizarse en períodos de encierro prolongado (Morales, 2020; Larraguibel et al., 2021).

En el caso de las relaciones con amistades o con pares, en casi todos los casos se señala que estas se han visto disminuidas por el hecho de la crisis sanitaria; sin embargo, manifiestan que existe un grupo pequeño, el más cercano, que se ha mantenido, dentro de los cuales hay amigas/os de la escuela.

A pesar de estas condicionantes en la vinculación entre pares, emergen algunas tácticas de escamoteo (De Certeau, 2000) que pretenden transgredir la formalidad dominante. Así, un joven de $3^{\circ}$ medio refiere que han creado un grupo aparte de las clases para jugar, reír, hablar de otros temas y no de materias escolares; otros crean prácticas de colaboración como tutoriales, guías, creación de una página para compartirla como vía para resolver la preocupación por las condiciones materiales de alguno/as compañeros/as. 
Ayudar a los alumnos que necesitan po, hacer rifa y todas esas cosas, lo mismo para el dieciocho se hicieron hartas actividades para poder recaudar dinero y ayudar a los otros compañeros po... en mercadería o los que necesitan ropa, o mismo las niñas que tienen hijos con ropas para sus bebés, pañales, leche. (E2, F, $3^{\circ}$ medio, RM, Recoleta)

Se identifican así acciones de solidaridad entre compañeros y compañeras de curso o colegio, relacionadas con necesidades materiales (colectas de mercaderías, rifas para recaudar dinero, etc.) o necesidades educativas (apoyo en desarrollo guías, elaboración de tutoriales, etc.). Se refleja la importancia de la escuela en el entramado relacional, pues no solo prima el proyecto educativo formal, sino que las relaciones políticas y socioafectivas que se desarrollan dentro de la institución, planteándose como relaciones políticas aquellas que cargan con la valoración de solidaridad. El fortalecimiento de la comprensión de la escuela como el espacio que contribuye al desarrollo personal — como se plantea más arriba- (Fariñas, 2005; Folardi et al., 2014), incluye por supuesto la y lo político, pues bajo los lentes freirianos los proyectos educativos de las escuelas se irán reproduciendo en este camino de desarrollo humano.

Respecto a la relación con las y los docentes destacan, sin embargo, que no existe mayor relación, salvo lo estrictamente vinculado con las clases. No es un tema que emergió como importante en las entrevistas, y las impresiones de las y los estudiantes no son unívocas; mientras señalan que algunos les ayudan, otros mencionan que complican la situación en la que se encuentran debido a la alta exigencia que tienen, sin considerar el contexto en el que viven. En esta misma línea, plantean que no hay una relación de confianza con la mayoría, y que a sus docentes mayores les cuesta manejarse con esta modalidad online. Aparece entonces un matiz importante en lo relacional, que sitúa un peso mayor en aquella experiencia que se da entre pares, y no tanto así con actores educativos adultos.

En cuanto a las experiencias emocionales en relación con esta modalidad de clases, de modo general, no son positivas; hay insatisfacción, frustración, 
disgusto con ella. Solo una estudiante señala que «no son tan malas» (E12, F, $1^{\circ}$ medio, RM, Lago Ranco); mientras que la única que refiere cierta satisfacción es una joven haitiana, para quien esta modalidad ha implicado asistir a clases online sin miedo, sin sentirse señalada o discriminada: «Así que las clases online es lo mejor (ríe), que me puede pasar, porque yo puedo hacer mis tareas en mi casa, sin miedo» (E10, F, $3^{\circ}$ medio, RM, Peñalolén), cuestión que sí experimentó las pocas semanas que alcanzó a asistir a clases presenciales, evidenciándose que los procesos educativos requieren entenderse también desde los momentos biográficos de cada estudiante y cómo experiencian a partir de ello estos procesos. La modalidad virtual de la educación aumentó la relevancia de esto, en tanto lo privado y los contextos vitales se vuelven más visibles y determinantes. Justamente, una de las dificultades señaladas se vincula con la mutación del espacio privado (hogar) en un espacio público destinado a responder con las distintas exigencias que deben enfrentar los grupos familiares, ya sea con labores educativas, teletrabajo, cuidados y trabajo doméstico. Esto redunda en que los límites entre una y otra labor sean difusos y simultáneos, derivando en ocasiones en tensiones y conflictos familiares.

Estudios señalan que la población juvenil (entre 12 y 29 años) ha experimentado variadas emociones que de cierta manera inciden en la salud mental; entre estas, se encuentran principalmente la sensación de miedo, tristeza e irritabilidad (Morales, 2020; Larraguibel et al., 2021; Andrades-Tobar, García, Valiente, Lucero, 2021). También se señala el estrés, principalmente por el encierro y el aislamiento (INJUV, 2020).

Finalmente, la información producida desde las entrevistas permite afirmar que la crisis surgida desde octubre de 2019 no impactó el comportamiento tradicional de sus aspiraciones, ni la percepción de lo que el mundo adulto espera de ellos. Así, estas se asocian a continuar estudios y entrar a la universidad, lo que tiene ciertas variaciones según estrato social - mantener 
privilegios en clases altas e ingresar al mundo universitario en clases medias y bajas para obtener mejores trabajos - (Duarte, Aniñir y Garcés, 2017; Castillo, 2019).

No obstante, a propósito de la crisis sociosanitaria, algunos jóvenes expresan dificultad para proyectar aspiraciones, evidencian incertidumbre, miedo al futuro y percepción de que las cosas van mal. Pero de modo general, respecto a las aspiraciones personales a corto plazo, la mayoría espera seguir estudiando, ya sea en la universidad o el liceo, según el momento biográfico actual de cada estudiante. A mediano plazo, en general, estudiantes esperan tener una profesión y algunos trabajar en un emprendimiento. En ese sentido, de manera transversal, la continuidad de estudios en la universidad es una aspiración. Entre los oponentes a la realización de las aspiraciones educativas y profesionales destacan las limitaciones económicas de las familias y las trabas burocráticas que tienen jóvenes migrantes para acceder a la gratuidad y otros beneficios. Quienes hacen referencia a factores ayudantes de cara a estas aspiraciones son, en todos los casos, mujeres y tiene que ver con actitudes personales (perseverancia, esfuerzo, sacrificio, optimismo) y apoyo familiar o de amistades del colegio.

Respecto a lo que esperan sus familias, encontramos diferencias en los/as estudiantes; mientras quienes están en cursos de enseñanza básica señalan que se espera que obtengan buenas calificaciones, en los cursos más altos emerge la expectativa a ser profesionales. En este último grupo, y particularmente en hombres, surge la contradicción entre lo que espera la sociedad, la presión social y los deseos personales.

Emergen también en las entrevistas otras metas u objetivos, que van desde la movilización social ascendente, el apoyo a la familia, hasta seguir profundizando y formándose a propósito de ciertos activismos políticos, tener nuevas amistades y actividades ocio-recreativas, como tomar clases de dibujo. Junto con ello, aparecen deseos relacionados con los efectos de la pandemia 
(sobre todo en los hombres), como volver a clases presenciales o realizar actividades que se han visto limitadas, como salir y pasarla bien con las amistades. Es decir, las aspiraciones de futuro trascienden la lógica de continuidad de estudios y de movilidad social, propiciadas por las movilizaciones estudiantiles y por la pandemia; si bien sigue siendo mayoritario el anhelo de continuidad de estudio, se complejiza debido a estas condiciones y agencias juveniles.

Respecto a prácticas de autocuidado, en general son mujeres quienes señalan haber comenzado a hacer alguna actividad nueva para sobrellevar la tensión del contexto de crisis social sanitaria y encierro. Se señalan actividades como hacer deporte, tejer, dibujar, ser autodidacta, gestionar redes online. Asimismo, un joven hombre señala haberse alejado de conflictos para estar mejor.

Algunos jóvenes señalan que les ha tocado cuidar a integrantes de su familia, principalmente hermanos/as más pequeño/as. Esto porque sus madres y padres trabajan de manera presencial u online, y deben ayudarlos.

También aparece en algunos estudiantes la participación en espacios sociales o escolares desde los cuales quieren aportar en cuidar a otros, así como también desde donde han recibido cuidados, en algunos casos puntuales desde profesores.

El análisis muestra cómo la dinámica relacional, si bien heterogénea durante la crisis, se ha visto afectada en las y los estudiantes, con una pérdida significativa del espacio entre pares y los efectos de ello en la salud mental. Al mismo tiempo, resulta relevante identificar las estrategias de enfrentamiento que van desde un fortalecimiento de las acciones de solidaridad y compromiso con las y los otros, hasta el aprendizaje de nuevas habilidades y herramientas. 


\section{CONCLusiones}

En el presente estudio hemos intentado aportar a la comprensión de las experiencias de las y los estudiantes de instituciones municipales y subvencionadas en el contexto de crisis sociosanitaria, buscando iluminar algunas reflexiones acerca de caminos de transformación para los tradicionales sistemas educativos.

Ello ha requerido al menos una doble aclaración inicial. La primera es dar cuenta de que esta crisis no se reduce únicamente a un problema sanitario o biomédico derivado por la pandemia global, sino que también se comprende en el marco de un profundo cuestionamiento al orden sociopolítico que vive el país desde las últimas décadas y que ha logrado hacerse más visible en Chile desde el 18 de octubre del 2019. Entenderlo de este modo, supone asumir que la experiencia educativa se encuentra condicionada a las múltiples desigualdades que operan en nuestra sociedad.

Una segunda aclaración se vincula a nuestro objeto de estudio. Si bien reconocemos la necesidad de atender a las transformaciones que sufren las instituciones educativas en el actual contexto, hemos hecho hincapié que ella requiere ser leída también desde las experiencias que construyen sus propios actores educativos, entendidos para nuestra investigación desde la multiplicidad de los mismos: apoderados, comunidad, directivos, docentes, estudiantes, etc. Así, la noción de experiencia en tanto herramienta teórica-conceptual posibilitó complejizar la mirada relevando algunos ejes de observación. Al respecto, las diversas respuestas que se han elaborado desde la política educativa y el modo en que los propios actores experiencian la educación en su realidad cotidiana evidencian un cierto desacoplamiento entre ambas.

De esta manera, los hallazgos obtenidos muestran que pese a los múltiples esfuerzos que se han realizado desde la normatividad institucional para responder 
al contexto, dichas respuestas no logran traducirse en las experiencias educativas de las y los estudiantes entrevistados/as. Es más, la revisión de antecedentes permitió analizar cómo la matriz adultocéntrica limita considerar a las y los estudiantes como actores sociales que pueden incidir en su propia experiencia educativa, pues tiende a primar una mirada que los reconoce principalmente como sujetos de protección y cuidado.

Visión contrapuesta a los hallazgos del presente estudio, toda vez que se observa cómo desde las propias experiencias estudiantiles se ejercen prácticas que permiten abrir rutas posibles para avanzar hacia la idea de otra escuela (Dussel, 2020). Lo anterior, no solo porque la crisis sociosanitaria mostró que efectivamente las instituciones educativas son posibles de ser transformadas a pesar de que presentan dificultades para una respuesta apropiada, sino que también porque la crisis ha revelado que la interacción directa resulta ser un aspecto irremplazable. De este modo, el vínculo socioemocional, la autonomía, la colaboración entre pares, el uso adecuado y pertinente de las tecnologías son algunas oportunidades para la transformación de la estructura educativa. No obstante, esto tiene como desafío considerar las experiencias estudiantiles en espacios de tomas de decisiones para hacer de la transformación de la escuela un lugar donde tengan cabida todos los actores educativos.

En cuanto a la experiencia, si bien en términos generales es significada como negativa, principalmente en términos de aprendizajes obtenidos, por las condiciones materiales con las que se cuentan y por las implicancias que ha conllevado este proceso para su bienestar socioemocional y en la interacción con otros. Este escenario adverso puede ser leído también en términos de desafíos para las comunidades educativas, pues deja de pensarse al estudiante solo desde el rol que cumple dentro de la institución educativa, dando paso a un entendimiento más complejo, que sitúa la experiencia y trayectoria educativas en ciertas condiciones sociales de existencia y donde los soportes institucionales se 
vuelven condición necesaria para garantizar universalmente el acceso y permanencia a la educación.

RECIBIDO: 9 DE JULIO DE 2021

ACEPTADO: 22 DE AGOSTO DE 2021

\section{BIBLIOGRAFÍA}

Andrades-Tobar, M., García, F., Valiente, C. y Lucero, C. (2021). Predictores de síntomas de ansiedad, depresión y estrés a partir del brote epidémico de COVID-19. Revista de Psicopatología y Psicología Clínica, 26(1), 13-22. https://doi.org/10.5944/rppc.28090

Appadurai, A. (2004). The Capacity to Aspire: Culture and the Terms of Recognition. En Culture and Public Action (pp. 59-84). Stanford University Press.

ArAujo, K. (2019). Hilos tensados: para leer el octubre chileno. Revista SulAmericana de Psicología, 8(1), 153-158. https://doi.org/10.29344/2318650x.1.2478

. (2009). Habitar lo social. Usos y abusos en la vida cotidiana en el Chile actual. Oxfam. LOM Ediciones.

Bourdieu, P. (1990). La juventud no es más que una palabra. Sociología y cultura, 7(2), 163-173.

Castillo, P. (2019). Subjetividad y dispositivo educacional chileno: desigualdad en discursos de jóvenes próximos a egresar de enseñanza media. En Juventudes en Chile. miradas de jóvenes que investigan. Volumen 2 (pp. 225-243). Santiago de Chile: Social-Ediciones.

CIAE (2020). COVID-19. Nuevos Contextos, Nuevas Demandas y Experiencia Docente en Chile. Recuperado de: http://eduinclusiva.cl/wpcontent/uploads/2020/10/CIAE-COVID-VFinal-1.pdf

Creswell, J. (2015). Educational Research: Planning, Conducting, and Evaluating Quantitative and Qualitative Research. Nueva York: Pearson.

Cuevas, H. y Budrovich, J. (2021). ¿Revolución, revuelta, despertar de un pueblo o «estallido social»? A un año de la crisis de octubre de 2019 en Chile.Revista F@ro, 2, 159-181. 
De Certeau, M. (2000). Valerse de usos y prácticas. En La invención de lo cotidiano 1. Artes de hacer (pp. 35-48). Universidad Iberoamericana/Instituto Tecnológico y de Estudios Superiores de Occidente.

Deleuze, G. (2006). Post-scriptum sobre las sociedades de control. Polis. Revista Latinoamericana, (13), 1-8. http://dx.doi.org/10.32735/S07186568/2006-N13-431

Díaz, C. y Navarro, P. (1995). Análisis de contenido. En J. M. Delgado y J. GUTIÉRREZ (eds.), Métodos y técnicas cualitativas de investigación en ciencias sociales (pp. 177-224). Madrid: Síntesis.

DuARTE, K. (2018). Genealogía del adultocentrismo. La constitución de un patriarcado adultocéntrico. En Juventudes en Chile. Miradas de jóvenes que investigan (pp. 17-47). Santiago de Chile: Social-Ediciones.

DuArte, K., AniÑIR, D. y Garcés, A. (2017). De encuentros y desencuentros entre jóvenes y personas adultas a propósito de salir de la enseñanza secundaria en Chile. Revista Latinoamericana de Ciencias Sociales, Niñez y Juventud, 15(1), 373-388.

DuBET, F. (2013). El declive de la institución. Profesiones, sujetos e individuos en la modernidad. Barcelona: Gedisa.

. (2011). La experiencia sociológica. Barcelona: Gedisa.

. (2010). Sociología de la experiencia. Madrid: Editorial Complutense y Centro de Investigaciones Sociológicas.

Dubet, F. y Martuccelli, D. (1997). En la escuela. Sociología de la Experiencia Escolar. Buenos Aires: Losada.

Dussel, I. (2020). La escuela en la pandemia. Reflexiones sobre lo escolar en tiempos dislocados. Práxis Educativa, 15, 1-16. https://doi.org/10.5212/PraxEduc.v.15.16482.090

FARIÑAS, G. (2005). Psicología, Educación y Sociedad. Ciudad de La Haban: Félix Varela.

FolADORI, H. et al. (2014). Aulear, hacia una pedagogía del acontecimiento. Santiago de Chile: Editorial Universitaria.

FoucAult, M. (2012). Vigilar y castigar: nacimiento de la prisión. Madrid: Biblioteca Nueva. 
FUNDACIÓN 99 (2020). Caracterización de la Educación Rural en Chile en contexto de pandemia por COVID-19. Recuperado de: https://fundacion99.org/

GARCÉS, M. (2020). Estallido social y una nueva Constitución para Chile. Santiago de Chile: LOM.

GONZÁlEZ, Y. y FeIXA, C. (2013). La construcción histórica de la juventud en América Latina. Bohemios, rockanroles y revolucionarios. Santiago de Chile: Cuarto Propio.

INJUV (2020). Sondeo: Salud mental COVID-19 población general 15 a 59 años. Recuperado de: https://www.injuv.gob.cl/sondeos

LAHIRE, B. (2004). Retratos sociológicos: disposições e variações individuais. Artmed Editora.

Larraguibel, Rojas-Andrade, M., Halpern, R. y Montt, M. (2021). Impacto de la Pandemia por COVID-19 en la Salud Mental de Preescolares y Escolares en Chile. Revista Chilena de Psiquiatría y Neurología de la Infancia y Adolescencia, 32(1), 12-21.

Madariaga, C. y Oyarce, A. M. (2020). Pandemia por COVID-19: Un hecho social total. Sus efectos sobre la salud mental de los chilenos. Ensayos sobre la pandemia Covid 19. Revista Chilena de Salud Pública, 13-29. https://doi:10.5354/0719-5281.2020.60371

MINEDUC (2021). Diagnóstico integral de aprendizaje. Recuperado de: https://www.meganoticias.cl/nacional/338013-diagnostico-integral-deaprendizajes-aprendizaje-emx01.html

. (2020a). Orientaciones Mineduc Covid-19. Recuperado de: https://www.mineduc.cl/wpcontent/uploads/sites/19/2020/03/OrientacionesMineduc_COVID19.pdf

. (2020b). Orientaciones generales para guiar el aprendizaje de los estudiantes a distancia. Recuperado de: https://educacionsuperior.mineduc.cl/wpcontent/uploads/sites/49/2020/04/Guia-aprendizajes-estudiantes-adistancia.pdf

. (2020d). Propuestas Mesa Técnica para la prevención de la Deserción Escolar. Recuperado de: https:/www.mineduc.cl/wpcontent/uploads/sites/19/2020/07/propuestas_desercionescolar.pdf . (2020e). Orientación al sistema escolar en contexto de COVID-19. Recueprado de: https://www.mineduc.cl/wpcontent/uploads/sites/19/2020/03/OrientacionesMineduc_COVID19.pdf 
- (2019). Orientaciones Contexto Nacional. Recuperado de: https://www.cpeip.cl/wpcontent/uploads/2019/11/OrientacionesContextoNacional.pdf

MORALES, C. (2020). Salud mental de los niños, niñas y adolescentes en situación de confinamiento. Revista anales, Séptima Serie (1), 303-318.

Martuccelli, D. y SANTiago, J. (2017). El desafio sociológico hoy. Individuo y retos sociales. Centro de Investigaciones Sociales.

Neut, P. (2015). La autoridad pedagógica desinvestida y la reconfiguración de sus legitimidades instituyentes. Revista de la Academia, 19, 59-96.

Pezo, H. (2019). ¿Liceo disciplinario?: condicionantes para la participación juvenil estudiantil. Una aproximación desde la perspectiva generacional. En Juventudes en Chile. Miradas de jóvenes que investigan. Volumen 2 (pp. 286-312). Santiago de Chile: Social-Ediciones.

Sepúlveda, L. y Valdebenito, M. J. (2014). ¿Las cosas claras? Aspiraciones de futuro y proyecto educativo laboral de jóvenes estudiantes secundarios. Estudios Pedagógicos (Valdivia), 40(1), 243-261.

Simons, M. y Masschelein, J. (2014). En defensa de la escuela. Una cuestión pública. Buenos Aires: Miño \& Dávila.

ScotT, J. (2001). La experiencia. La Ventana, 13, 42-73.

Strauss, A. y CoRBIN, J. (2002). Bases de la investigación cualitativa-técnicas y procedimientos para desarrollar la teoría fundamentada. Editorial Universidad de Antioquia.

TRINDADE, V. A. (2014). Entrevistando en investigación cualitativa y los imprevistos en el trabajo de campo: de la entrevista semiestructurada a la entrevista no estructurada. En P. SCHETTINI e I. CORTAZzo, Técnicas y estrategias en la investigación cualitativa (pp. 18-34). La Plata: Universidad Nacional de la Plata.

Vygotski, L. (1987). Historia del desarrollo de las funciones psíquicas superiores. La Habana: Científico-Técnica. 